\title{
Reconstrucción de pared torácica con material de fijación en pacientes con lesiones tumorales. Serie de casos
}

\author{
Reconstruction of the chest wall with fixation in patients with \\ tumor lesions. Case series
}

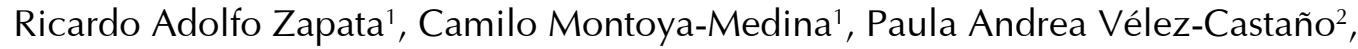 Lennis Bedoya-Muñoz ${ }^{3}$

\begin{abstract}
Médico, especialista en Cirugía general, subespecialista en Cirugía de tórax, clínica CardioVID, Medellín, Colombia. Médica, residente de Cirugía general, Universidad Militar Nueva Granada, Bogotá, D.C., Colombia.

Médica, Servicio de Cirugía de tórax, Clínica CardioVID, Medellín, Colombia
\end{abstract}

\section{Resumen}

Introducción. El manejo quirúrgico de las lesiones de la pared costal con resección y reconstrucción con material protésico ha venido en aumento, debido al avance en las técnicas quirúrgicas y en la experiencia del cirujano, así como en la innovación y las características de los materiales protésicos, que permiten realizar resecciones amplias de la pared garantizando una mayor estabilidad esquelética, una menor alteración de la mecánica respiratoria y mejores los resultados estéticos.

Métodos. Se presenta la experiencia en la clínica CardioVID, Medellín, Colombia, entre los años 2015 y 2019 , mediante una revisión retrospectiva de 8 casos sometidos a resección de lesiones benignas de la pared torácica y reconstrucción con material protésico.

Resultados. Se encontró una adecuada evolución de los pacientes, con un buen resultado estético y funcional, con adecuado control de la enfermedad, sin reportes de complicaciones ni de recidiva en el seguimiento.

Discusión. Una técnica adecuada permite la reconstrucción de estos defectos con complicaciones mínimas y bajas tasas de extracción de prótesis, finalizando con excelentes resultados funcionales y cosméticos.

Con nuestra experiencia podemos concluir que la elección adecuada de los pacientes candidatos a manejo quirúrgico, una buena técnica quirúrgica y un personal con experiencia son cruciales para lograr buenos resultados en cuanto a función pulmonar y estética. Además de lograr una sobrevida bajo los parámetros establecidos posterior a lograr una resección R0.

Palabras clave: pared torácica; neoplasias; cirugía torácica; reconstrucción; prótesis e implantes; titanio.

Fecha de recibido: 22/02/2020 - Fecha de aceptación: 07/04/2020

Correspondencia: Paula Andrea Vélez-Castaño, Calle 13 sur \# 8-90, Bogotá, D.C., Colombia. Teléfono: 3014028096

Correo electrónico: pau.velca@gmail.com

Citar como: Zapata RA, Montoya-Medina C, Vélez-Castaño PA, Bedoya-Muñoz L. Reconstrucción de pared torácica con material de fijación en pacientes con lesiones tumorales. Serie de casos. Rev Colomb Cir. 2021;36:66-73. https://doi.org/10.30944/20117582.545

Este es un artículo de acceso abierto bajo una Licencia Creative Commons - BY-NC-ND https://creativecommons.org/licenses/by-ncnd/4.0/deed.es 


\begin{abstract}
Introduction. Surgical management of chest wall lesions with resection and reconstruction with prosthetic material has been increasing, due to advances in surgical techniques and surgeon's experience, as well as innovation and characteristics of prosthetic materials. They allow wide resections of the wall, guaranteeing greater skeletal stability, less alteration of respiratory mechanics, and better aesthetic results.
\end{abstract}

Methods. The experience in the CardioVID clinic, Medellín, Colombia, between the years 2015 and 2019, is presented through a retrospective review of eight cases that underwent resection of benign lesions of the chest wall and reconstruction with prosthetic material.

Results. An adequate evolution of the patients was found, with a good aesthetic and functional result, with adequate control of the disease, without reports of complications or recurrence in follow-up.

Discussion. An adequate technique allows the reconstruction of these defects with minimal complications and low prosthesis extraction rates, ending with excellent functional and cosmetic results. With our experience, we can conclude that the proper choice of patients who are good candidates for surgical management, a good surgical technique, and an experienced staff are crucial to achieve good results in terms of lung function and aesthetics results, in addition to obtain survival under the established parameters after achieving an R0 resection.

Keywords: thoracic wall; neoplasms; thoracic surgery; reconstruction; prostheses and implants; titanium.

\section{Introducción}

La resección y reconstrucción de la pared costal es un tema ampliamente debatido dadas las opciones quirúrgicas que hay en la actualidad, la evolución en la técnica, en los materiales protésicos y en las terapias oncológicas desarrolladas, lo que ha permitido aumentar las indicaciones quirúrgicas para la resección de las lesiones costales y su posterior reconstrucción con materiales protésicos. Sin embargo, en manos sin la suficiente experiencia sigue siendo un procedimiento con alta morbilidad, alta tasa de complicaciones postoperatorias, e incluso, alta mortalidad ${ }^{1}$.

La mayoría de los procedimientos de reconstrucción son secundarios a la resección quirúrgica de tumores malignos, ya sea en un intento curativo o como parte del manejo paliativo; pudiéndose realizar prácticamente en cualquier estructura anatómica de la pared torácica, dependiendo del resultado histopatológico, pronóstico y posibilidad de resección completa (R0).

Los tumores de la pared costal son tumores raros, que representan el $0,04 \%$ de los nuevos casos de cáncer y el $5 \%$ de las neoplasias del tórax. El 50 \% son benignos. Los tumores de la pared torácica pueden ser primarios, metastásicos o por extensión. De los tumores malignos primarios de pared, el $55 \%$ surgen del hueso o cartílago, y el $45 \%$ de tejidos blandos. Las metástasis óseas en la caja torácica usualmente son secundarias a cáncer de pulmón, mama o próstata ${ }^{2-4}$.

En la literatura se ha descrito que los principales factores que influyen en la sobrevida de los pacientes son la resección completa del tumor (R0), la histología y el grado de diferenciación del tumor ${ }^{5}$. El pronóstico del tratamiento quirúrgico depende de la resección completa (R0), del estadio clínico del tumor y de su estirpe etiológica. Se debe tener en cuenta que los pacientes que tienen invasión de la pared torácica por tumores pulmonares, con ausencia de afectación ganglionar y metastásica, se benefician de la resección quirúrgica en bloque ${ }^{6}$. Si la etiología es tumoral, los márgenes de resección adecuados deben ser mínimo de $4 \mathrm{~cm}$ para los tumores malignos y de 2 $\mathrm{cm}$ para las metástasis o para tumores benignos. Si se trata de un proceso infeccioso, el límite de resección estará determinado por la viabilidad de los tejidos al efectuar el desbridamiento ${ }^{7}$.

En los pacientes con neoplasia pulmonar de células no pequeñas que infiltra la pared costal, se debe garantizar una enfermedad N0 - N1 ya 
que los pacientes N2 no presentan mejoría en la sobrevida. Además, se consideró que el parámetro $\mathrm{T}$ es menos relevante para el pronóstico, siempre y cuando la resección sea completa, con los márgenes adecuados. La supervivencia a 5 años de estos pacientes con diagnóstico de neoplasia pulmonar de células no pequeñas puede varían entre el 28 al $61 \%{ }^{8}$.

Hay múltiples opciones de reconstrucción de la pared costal y ninguno de los métodos cumple con todas las características, necesarias o ideales para la reconstrucción, por lo cual la elección depende de cada paciente y de la experiencia del grupo quirúrgico. Sin embargo, hay recomendaciones sobre el uso de prótesis rígidas en pacientes con defectos grandes de la pared, que se encuentren sobre las áreas críticas del tórax, definidas como la pared anterior y lateral ${ }^{9,10}$.

Además de los avances en la técnica quirúrgica y los materiales protésicos, el manejo en conjunto con Cirugía plástica ha proporcionado mejoría en el pronóstico, al combinarse la reconstrucción de la pared con los diferentes colgajos musculocutáneos, logrando mejores resultados en la estabilidad de la pared, la función pulmonar y el resultado estético final, sin verse afectada significativamente por el tamaño de la resección o la ubicación de la lesión ${ }^{2}$.

\section{Métodos}

Se hizo una revisión retrospectiva de la historia clínica e imágenes diagnósticas de los pacientes sometidos a resección de lesiones de pared torácica y reconstrucción con material protésico por lesiones benignas, para presentar la experiencia en la clínica CardioVID, en Medellín, Colombia, entre los años 2015 y 2019.

\section{Resultados}

A continuación, se presentan los casos clínicos de los ocho pacientes operados por los Cirujanos de tórax de la clínica CardioVID, en Medellín, Colombia, en quienes se practicó resección de lesión de pared torácica y reconstrucción con material protésico.

Caso 1. Se trata de una paciente de sexo femenino de 48 años de edad, sin antecedentes médicos ni quirúrgicos de importancia, que ingresó con un cuadro de dolor torácico tipo pleurítico derecho de varios meses de evolución. Por tomografía computarizada (TC) se identificó una masa en el lóbulo superior del pulmón derecho, con compromiso pleural, en íntimo contacto con los arcos costales y vértebras a nivel de T3 y T4. Se hizo diagnóstico histopatológico con la biopsia guiada por TC de neoplasia fusocelular. En la resonancia magnética nuclear (RMN) se halló una lesión que comprometía la vaina neural periférica en T3 y T4, compatible con Schwanoma. Se llevó a cirugía para resección y reconstrucción de pared del tórax, con abordaje conjunto con neurocirugía para discectomía cervical, exploración y descompresión del canal raquídeo por laminectomía, resección de la lesión de mediastino posterior más resección de pared torácica del tercer y cuarto arco costal, con un defecto en la pared de $5 \times 5 \mathrm{~cm}$. Se hizo reconstrucción de la pared con sistema de clips y barras de conexión de titanio. La patología confirmó un Schwannoma con resección R0. Durante el seguimiento con adecuada evolución clínica, radiografía de tórax con barras en adecuada posición, sin alteraciones en la funcionalidad de la paciente, ni recidiva de la enfermedad.

Caso 2. Paciente masculino de 49 años de edad, que presentó cuadro clínico de varios meses de evolución de lesión palpable en la reja costal izquierda, con aumento progresivo del tamaño y dolor asociado, sin síntomas constitucionales. La TC de tórax evidencia una lesión sólida de 3 × 3 $\mathrm{cm}$, en pared torácica posterior izquierda, entre octavo y noveno espacio intercostal. Se resecó el tumor, quedando un defecto de la pared de 5 × 6 $\mathrm{cm}$. Se realizó reconstrucción con malla de Prolene $®$ y dos barras de titanio de fijacion en "U" con tornillos bloqueados. La patología reportó mixoma celular intramuscular. El seguimiento clínico y tomográfico son adecuados, con buena evolución clínica e imagenológica y sin evidencia de recidiva de la enfermedad.

Caso 3. Paciente masculino de 22 años de edad, que presentó tumor costocondral y dolor torácico, con sospecha de encondroma, por lo que fue llevado a resección de lesión, con un tamaño total 
del defecto de 4 x $4 \mathrm{~cm}$. Se hizo reconstrucción torácica con malla de Prolene ${ }^{\circledR}$ y colocación de barras de titanio de fijacion en " $U$ " con tornillos bloqueados a nivel de séptimo y octavo arco costal anterior derecho. La patología confirmó un encondroma con márgenes libres. Sin seguimiento posterior por la especialidad.

Caso 4. Paciente de sexo masculino de 67 años de edad, que ingresó al servicio con hallazgo incidental de lesión de tipo tumoral en séptima costilla izquierda a nivel posterior durante realización de angioTC para estudio por electrofisiología por flutter atrial. Con sospecha diagnóstica de encondroma versus osteosarcoma, se llevó a biopsia guiada por $\mathrm{TC}$, con resultado sugestivo de hemangioma capilar. El PET TC evidenció una lesión sin aumento del metabolismo. Se llevó a cabo resección de la lesión del séptimo arco costal, con un defecto total de $5 \times 5 \mathrm{~cm}$, y reconstrucción con malla de Prolene ${ }^{\circledR}$ y una barra de titanio de fijacion en "U" con tornillos bloqueados (figura 1). Resultado final de patología concordante con hemangioma intraóseo. Al año de la intervención presentó caída desde su propia altura con fractura de barra de titanio por lo cual se llevó a procedimiento quirúrgico para extracción de la barra. El seguimiento posterior sin alteraciones en la funcionalidad del paciente ni en pared torácica, sin recidiva de la lesión a la fecha.

Caso 5. Paciente masculino de 59 años, quien presentó cuadro clínico de varios meses de evolución de lesión expansiva en la región lateral derecha del tórax, asociada a dolor, sin síntomas constitucionales. Se hizo diagnóstico histopatológico por biopsia guiada por TC de neoplasia mesenquimal fusiforme de bajo grado, compatible con fibromatosis de tipo desmoide (figura 2). Se llevó a resección de la lesión con extracción de noveno y décimo arco costal, con un tamaño total del defecto de $6 \times 8 \mathrm{~cm}$, con reconstrucción con dos barras de titanio de fijacion en "U" con tornillos bloqueados y malla de Prolene $®$. La patología confirmó la fibromatosis de tipo desmoide con márgenes libres. En seguimiento con adecuada evolución y sin recidiva de la enfermedad en la última valoración.

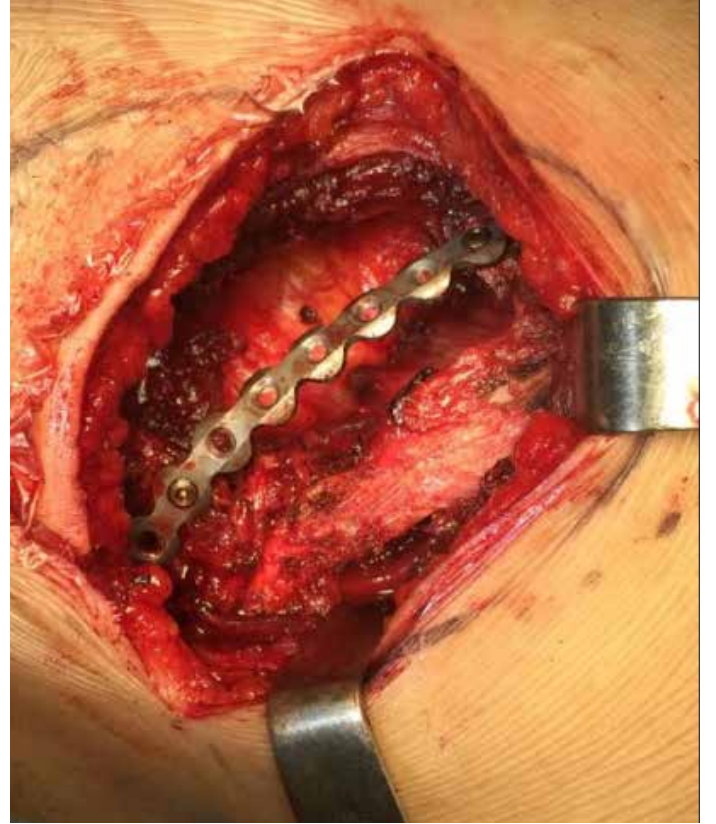

Figura 1. Caso 4. Paciente sometido a resección de un arco costal y reconstrucción con una barra de titanio.

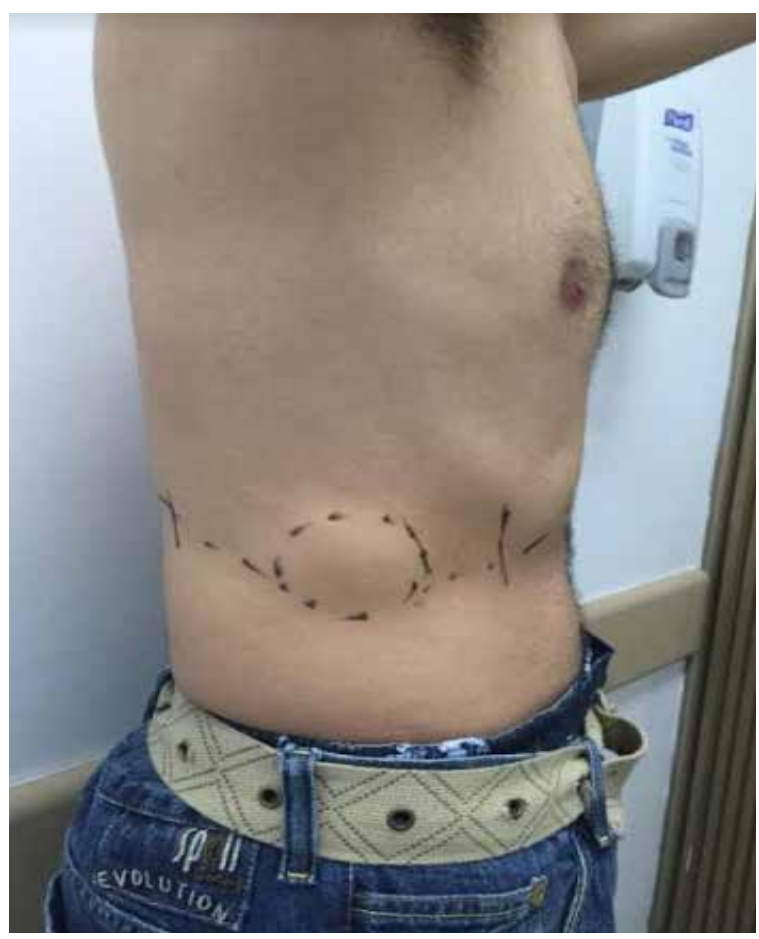

Figura 2. Caso 5. Paciente programado para resección de la lesión con extracción de noveno y décimo arco costal con reconstrucción con dos barras de titanio. Resultado de patología confirma fibromatosis de tipo desmoide. 
Caso 6. Paciente de sexo masculino de 38 años de edad, que ingresó remitido por un cuadro clínico de dolor en hemitórax derecho de un año de evolución. Se practicó tomografía de tórax en la que se encontró una lesión en la parte lateral del noveno arco costal derecho, de aspecto lítico, con adelgazamiento de la cortical, y gammagrafía ósea, con aumento de la captación. Se llevó a biopsia por trucut con histología compatible con displasia fibrosa. Se practicó resección de lesión de noveno arco costal, con un defecto de la pared de $4 \times 3 \mathrm{~cm}, \mathrm{y}$ reconstrucción con una barra de titanio de fijacion en "U" con tornillos bloqueados y malla de Prolene ${ }^{\circledR}$ (figura 3). La patología confirmó el diagnóstico de displasia fibrótica, con resección incompleta (R1). El paciente no asistió a seguimiento para control.

Caso 7. Paciente masculino de 60 años de edad, que consultó por un cuadro clínico de varios meses de evolución de dolor en hemitórax izquierdo. La TC de tórax mostró una lesión lítica en el séptimo arco costal lateral izquierdo, con engrosamiento pleural y fractura patológica. La biopsia guiada por TC fue negativa para malignidad y sospechosa de encondroma. Se llevó a resección quirúrgica con un defecto de $3 \times 4 \mathrm{~cm}$ y reconstrucción con malla de Prolene ${ }^{\circledR}$ y barra de titanio de fijacion en " $U$ " con tornillos bloqueados a nivel de séptimo arco costal lateral. El resultado de patología confirmó el diagnóstico de enfermedad de Erdheim-Chester, con resección completa. Los seguimientos son adecuados, sin recidiva de la enfermedad.

Caso 8. Mujer de 66 años, quien ingresa con un cuadro de varios meses de evolución de tos seca irritativa, asociada a dolor torácico inespecífico. Los estudios de imagen muestran una masa en la pared torácica, a nivel de séptimo arco costal derecho, en la región lateral, con una fractura asociada. Se llevó a resección de lesión con sexto, séptimo y octavo arco costal lateral derecho, con un defecto de pared de $7 \times 8 \mathrm{~cm}$, con reconstrucción posterior con 3 barras de titanio de fijacion en "U" con tornillos bloqueados y malla de Prolene ${ }^{\circledR}$. El resultado de la patología reportó hemangioma intraóseo, con resección completa. Durante el seguimiento presenta adecuada evolución y funcionalidad, sin recidiva de la enfermedad.

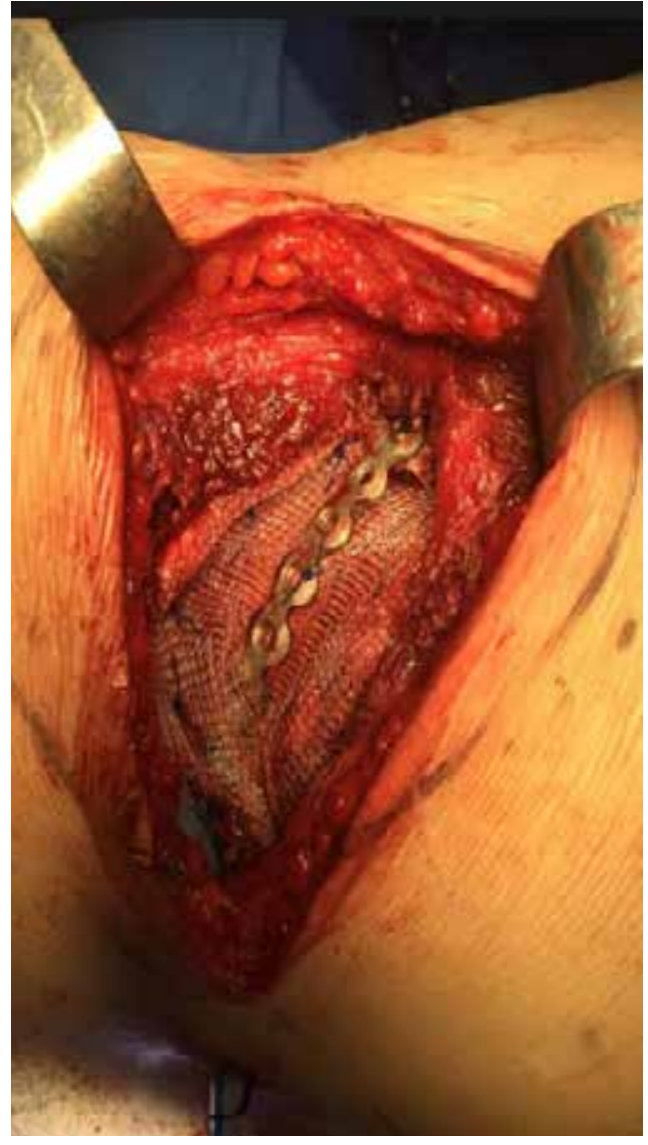

Figura 3. Caso 6. Paciente en quien se practicó resección de un arco costal y reconstrucción con una barra de titanio y refuerzo con malla de Prolene ${ }^{\circledR}$.

\section{Discusión}

La resección de las lesiones de la pared torácica con reconstrucción con prótesis ha venido en aumento, convirtiéndose en una opción paliativa y curativa, tanto para lesiones malignas como benignas. Sin embargo, algunas de estas resecciones a menudo repercuten en grandes defectos de la pared torácica, lo que puede conducir a inestabilidad esquelética, alteraciones de la mecánica respiratoria y defectos cosméticos significativos ${ }^{11 .}$

El objetivo principal del tratamiento quirúrgico es la resección del tumor con márgenes microscópicamente negativos, resección R0, garantizando así una resección oncológica adecuada ${ }^{12-14}$, además de otros objetivos secundarios para la reconstrucción de la pared torácica como ${ }^{11 .}$ : 
- Restauración de la rigidez de la pared torácica.

- Prevención de una hernia pulmonar.

- Evitar la contracción de la pared torácica.

- Prevención del atrapamiento de la escápula (cuando la resección involucra las costillas 5 y 6).

- Proteger los órganos mediastínicos subyacentes.

- Buen resultado cosmético.

Existen indicaciones quirúrgicas para la reconstrucción de la pared costal, por lo que cada paciente debe ser individualizado y evaluado detenidamente. En general, se considera que las resecciones que involucran 2 costillas o menos, no requerirían estabilización de la pared torácica, sino simplemente la reconstrucción de los tejidos blandos para cerrar el defecto ${ }^{15}$. La literatura menciona que los defectos más grandes o con necesidad de resección de 4 o más costillas, en la cara lateral o anterior, generalmente requerirán reconstrucción esquelética con materiales biológicos, aloplásicos o sintéticos.

Además, es importante la ubicación del defecto, puesto que los defectos ubicados debajo de la escápula, adyacentes a la columna o al músculo pectoral mayor anterior generalmente no requieren prótesis, ya que tienen una adecuada cobertura y rigidez suficientes para garantizar la mínima alteración en la mecánica ventilatoria. Pero defectos laterales o inferoanteriores, junto con las resecciones del esternón, generalmente requerirán una reconstrucción protésica para garantizar, tanto la estabilidad, como la cobertura de estructuras intratorácicas ${ }^{16}$ (figura 2).

Teniendo en cuenta lo anterior, el enfoque quirúrgico para la reconstrucción de la pared torácica se puede dividir en 2 fases, realizadas en cirugía de una etapa ${ }^{11}$ :

- Restauración de la integridad esquelética.

- Cobertura del defecto con tejidos blandos

Existen múltiples técnicas y materiales para la reconstrucción de la pared torácica, como malla sintética, materiales bioprotésicos y sistemas de osteosíntesis, sin embargo, en esta revisión nos centramos en el uso de barras de titanio en combinación con malla de polipropileno.

Los sistemas de osteosíntesis son sistemas de base metálica que se utilizan para unir múltiples costillas y / o defectos en el esternón, los cuales permiten un mayor movimiento fisiológico de las costillas, comparado con el uso de malla. El sistema de osteosíntesis más comúnmente usado es a base de titanio, dada su mayor resistencia a la tracción y a la infección ${ }^{11}$ (figuras 1 y 3 ).

Uno de los puntos más importantes a favor de los sistemas protésicos es su facilidad de implantación y su seguimiento relativamente libre de complicaciones. Algunas de estas, que se presentan en un bajo porcentaje, es la fractura de la barra, que puede variar entre 0-11 \% ${ }^{17}$. El titanio actual posee una gran resistencia, sin embargo, los estudios han demostrado que la tensión repetida en el metal puede iniciar y propagar microgrietas, que predisponen a una falla total por fractura de la barra. A pesar de haber un porcentaje bajo de falla de la barra, esta ocurre principalmente en las reconstrucciones de pared torácica anterior, debido al movimiento del tórax durante la respiración y el estrés repetido. La extracción quirúrgica comúnmente se requiere después de una falla por fractura de la barra, ya que condiciona dolor, así como también el riesgo de lesión por migración del implante ${ }^{18}$.

En este artículo se presenta una serie de ocho casos de resección de lesión de pared costal con reconstrucción posterior con barras de titanio, y en algunos casos con malla de polipropileno, todos ellos con reporte de patología de lesiones benignas. Solo hubo un caso de resección incompleta y un reporte de fractura de una barra, por caída del paciente desde su propia altura, con necesidad de reoperación para retiro del implante a los 19 meses. No se detectaron otras complicaciones, como infecciones del sitio operatorio ni rechazos del material protésico.

\section{Conclusiones}

La reconstrucción de la pared torácica con protesis de titanio es un procedimiento seguro, con 
buena tolerancia y con una baja tasa de complicaciones postoperatorias, como podemos observar en los casos descritos en nuestra serie. La mejoría de la técnica quirúrgica y la experiencia del grupo quirúrgico favorecen los buenos resultados estéticos, la buena función pulmonar y la pronta reincorporación a la vida laboral, con una rápida recuperación en el postoperatorio y menor estancia hospitalaria.

\section{Cumplimiento de normas éticas}

Consentimiento informado. Según la Resolución 8430 de 1993, del Ministerio de Salud de Colombia, esta investigación se clasifica como estudio sin riesgo pues consiste en una revisión retrospectiva de historias clínicas, por lo que no se requiere diligenciamiento del consentimiento informado. El estudio fue aprobado por el Comité de ética institucional de la clínica.

Conflicto de interés. Los autores declaramos que no hay conflicto de intereses ni plagios. No hemos conferido ningún derecho o interés a terceras personas.

Financiación. No existe financiación externa alguna para la elaboración de este estudio.

\section{Contribución de los autores.}

Diseño: Ricardo Zapata.

Adquisición de datos, análisis e interpretación de datos y redacción del manuscrito: Paula Vélez.

Revisión crítica: Ricardo Zapata, Camilo Montoya, Lennis Bedoya.

\section{Referencias}

1. Spicer JD, Shewale JB, Antonoff MB, Correa AM, Hofstetter WB, Rice DC, et al. The influence of reconstructive technique on perioperative pulmonary and infectious outcomes following chest wall resection. Ann Thorac Surg. 2016;102:1653-9. https://doi.org/10.1016/j.athoracsur.2016.05.072

2. Harati K, Kolbenschlag J, Behr B, Goertz O, Hirsch T, Kapalschinski N, et al. Thoracic wall reconstruction after tumor resection. Front Oncol. 2015;5:1-10. https://doi.org/10.3389/fonc.2015.00247

3. Shah AA, D’Amico TA. Primary chest wall tumors. J Am Coll Surg. 2010;210:360-6. https://doi.org/10.1016/j.jamcollsurg.2009.11.012
4. David EA, Marshall MB. Review of chest wall tumors: a diagnostic, therapeutic, and reconstructive challenge. Semin Plast Surg. 2011;25:16-24. https://doi.org/10.1055/s-0031-1275167

5. Foroulis CN, Kleontas AD, Tagarakis G, Nana C, Alexiou I, Grosomanidis V, et al. Massive chest wall resection and reconstruction for malignant disease. Onco Targets Ther. 2016;9:2349-58. https://doi.org/ 10.2147/OTT.S101615

6. Palafox D, Palafox J. Resección tumoral en bloque y reconstrucción de la pared torácica. Cir plást iberolatinoam. 2011;37:239-42.

7. Lasso JM, Uceda M, Arenas L, Pérez-Cano R. Reconstrucción de defectos torácicos de espesor total. Presentación de 8 casos de especial complejidad. Cir plást iberolatinoam. 2009;35;249-60.

8. Filosso PL, Sandri A, Guerrera F, Solidoro P, Bora G, Lyberis $\mathrm{P}$, et al. Primary lung tumors invading the chest wall. J Thorac Dis. 2016;8:S855-62.

https://doi.org/10.21037/jtd.2016.05.51

9. Scarnecchia E, Liparulo V, Capozzi R, Ceccarelli S, Puma $\mathrm{F}$, Vannucci J. Chest wall resection and reconstruction for tumors: analysis of oncological and functional outcome. J Thorac Dis. 2018;10(suppl. 16):S1855-63. https://doi.org/10.21037/jtd.2018.05.191

10. Puma F, Vannucci J. Chest wall resection/reconstruction for tumors. In: Mathisen DJ, Morse CR, Fischer JE, editors. Master Techniques in Surgery. Thoracic Surgery. Philadelphia: Wolters Kluwer; 2015. p. 312-58.

11. Khullar OV, Fernandez FG. Prosthetic reconstruction of the chest wall. Thorac Surg Clin. 2017;27:201-8. https://doi.org/10.1016/j.thorsurg.2017.01.014

12. Lehnhardt M, Hirche C, Daigeler A, Goertz O, Ring A, Hirsch T, et al. Soft tissue sarcoma of the upper extremities. Analysis of factors relevant for prognosis in 160 patients. Chirurg. 2012;83:143-52. https://doi.org/10.1007/s00104-011-2124-6

13. Daigeler A, Zmarsly I, Hirsch T, Goertz O, Steinau HU, Lehnhardt M, Harati K. Long-term outcome after local recurrence of soft tissue sarcoma: a retrospective analysis of factors predictive of survival in 135 patients with locally recurrent soft tissue sarcoma. Br J Cancer. 2014;110:1456-64. https://doi.org/10.1038/bjc.2014.21

14. Kandel R, Coakley N, Werier J, Engel J, Ghert M, Verma $\mathrm{S}$, and the Sarcoma Disease Site Group of Cancer Care Ontario's Program in Evidence-Based Care. Surgical margins and handling of soft-tissue sarcoma in extremities: a clinical practice guideline. Curr Oncol. 2013;20:e247-54.

https://doi.org/10.3747/co.20.1308 
15. Leuzzi G, Nachira D, Cesario A, Novellis P, Petracca-Ciavarella $\mathrm{L}$, Lococo F, et al. Chest wall tumors and prosthetic reconstruction: a comparative analysis on functional outcome. Thorac Cancer. 2015;6:247-54. https://doi.org/10.1111/1759-7714.12172

16. Ferraro $P$, Cugno S, Liberman M, Danino MA, Harris PG. Principles of chest wall resection and reconstruction. Thorac Surg Clin. 2010;20:465-73.

https://doi.org/10.1016/j.thorsurg.2010.07.008
17. Berthet JP, Canaud L, D'Annoville T, Alric P, Marty-Ane CH. Titanium plates and Dualmesh: a modern combination for reconstructing very large chest wall defects. Ann Thorac Surg. 2011;91:1709-16. https://doi.org/10.1016/j.athoracsur.2011.02.014

18. Miller DL, Force SD, Pickens A, Fernandez FG, Luu T, Mansour KA. Chest wall reconstruction using biomaterials. Ann Thorac Surg. 2013;95:1050-6. https://doi.org/10.1016/j.athoracsur.2012.11.024 